This item was submitted to Loughborough's Research Repository by the author.

Items in Figshare are protected by copyright, with all rights reserved, unless otherwise indicated.

\title{
Coordinated multicell beamforming with local and global data rate constraints
}

PLEASE CITE THE PUBLISHED VERSION

http://dx.doi.org/10.1109/EUSIPCO.2016.7760476

PUBLISHER

(c) IEEE

VERSION

AM (Accepted Manuscript)

\section{PUBLISHER STATEMENT}

This work is made available according to the conditions of the Creative Commons Attribution-NonCommercialNoDerivatives 4.0 International (CC BY-NC-ND 4.0) licence. Full details of this licence are available at: https://creativecommons.org/licenses/by-nc-nd/4.0/

\section{LICENCE}

CC BY-NC-ND 4.0

\section{REPOSITORY RECORD}

Elsabae, Ramadan G.M., Bokamoso Basutli, Kinan Ghanem, Yu Gong, and Sangarapillai Lambotharan. 2019. "Coordinated Multicell Beamforming with Local and Global Data Rate Constraints". figshare.

https://hdl.handle.net/2134/24173. 


\title{
Coordinated Multicell Beamforming with Local and Global Data Rate Constraints
}

\author{
Ramadan Elsabae, Bokamoso Basutli, Kinan Ghanem, Yu Gong, and Sangarapillai Lambotharan \\ Signal Processing and Networks Research Group \\ Loughborough University \\ Loughborough, Leicestershire, UK, LE11 3TU \\ Email: R.G.M.Elsabae, B.Basutli, K.Ghanem, Y.Gong, S.Lambotharan\{@1boro.ac.uk\}
}

\begin{abstract}
This paper outlines how Singular Value Decomposition (SVD) technique can be used to simplify a multicell network with heterogenous users. The heterogenous users considered in this work comprise of single antenna local users and one multiantenna global user. The local users are attached to their respective base stations (BSs) while the global user is jointly served by multiple BSs. We consider downlink beamforming design using power minimization approach. It is shown that the data rate to the global user should be split equally amongst the serving BSs.
\end{abstract}

Index Terms-Beamforming, SVD, downlink, SINR target, multiantenna, quality of service.

\section{INTRODUCTION}

With technology trends of today, where wireless networks have data hungry users, it is necessary to consider cell densification that enhances frequency reusage [1], [2]. Multiantenna deployment at both mobile users and BSs also enable the mobile network to take advantage of the spatial diversity in order to increase the overall performance of the network. Various coordinated beamformer techniques have been developed for downlink beamforming in multiantenna wireless systems [3]-[6]. The use of generalized singular value decomposition (GSVD) for coordinated beamforming in MIMO system was examined in [7]. In [8], a multiuser multiinput multi-output (MU-MIMO) network was considered. The work in [8] showed that by introducing a limited number of zero-forcing constraints, the SINRs of all stream are decoupled and this reduces the problem to a multiuser multi-input singleoutput (MU-MISO) problem. The setback of this approach is the reduced degrees-of-freedom and inefficiency. Coordinated beamforming design with weighted power minimization was considered in [9] using Lagrangian duality theory. The work in [10] considered power minimization problem in a network wherein users are served by joint non-coherent multiflow beamforming. The authors in [10] emphasized that even though the users can be served by multiple transmitters, the information symbols are coded and transmitted independently. Coordinated beamforming with user fairness based SINR balancing techniques were also considered in [11]-[16].

In this paper, we aim to study joint downlink beamforming using power minimization approach. The users have known specific data rate targets that need to be satisfied. We consider optimal solution that meets the target SINRs for all users.

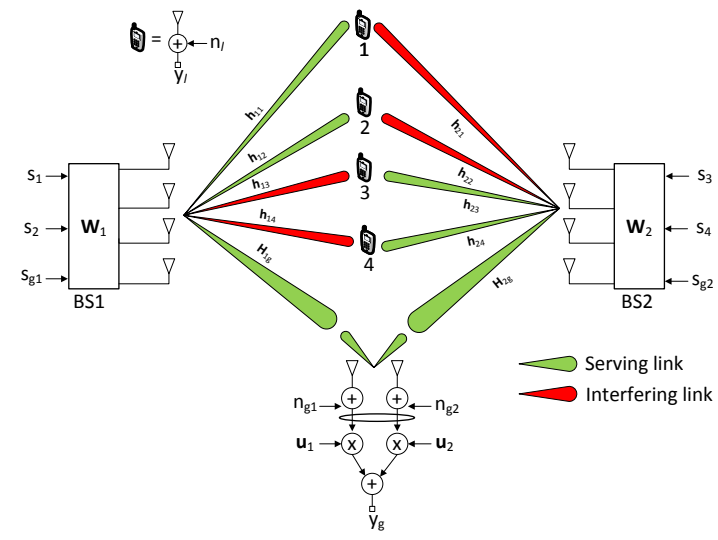

Fig. 1. Network topology. Both BS1 and BS2 serve two local user and one global user.

The set of users considered in this work consist of single antenna local users and a single multiantenna global user. The global user is served by multiple BSs. By using SVD, we decompose the multi-input multi-output (MIMO) channels between the BSs and the global user to form parallel and independent multi-input single-output (MISO) channels. The approach requires no phase synchronization between the BSs that are serving the global user. According to the proposed scheme, certain users are served by only a single base station (BS), however, one multiantenna terminal is served by two BSs. The latter user is known as global user which receives data from both BSs simultaneously. Hence, optimum split of data rate from different BS is also considered in this paper. Even though one global user is considered in this paper, it is possible to extend our work to multiple global users who can benefit from different channel conditions of both the BSs, especially when the users are at the cell edge.

The work is organized as follows: Section II presents the system model and assumptions; also it shows the mathematical framework and highlights the problem formulation. SVD based beamforming design is demonstrated in section III. Numerical Performance Analysis is conducted in section IV. Finally, the discussions are concluded in Section V.

\section{System Model and Assumptions}

This paper considers a network comprising of two basestations denoted $n=[1,2]$ as depicted in Figure 1. Each BS 
is equipped with $M$ antennas and it serves $L_{n}$ single antenna local users in the cell $n$. We denote a set of all local users and all BSs as $\mathcal{L}$ and $\mathcal{N}$ respectively. There is a global user denoted $g$ being served by both BSs. The global user is equipped with two antennas. We assume that all the BSs operate in the same frequency and that all users experience considerable intercell interference.

\section{A. Problem Formulation}

In the downlink, the transmitted signal for $l$-th local user from $n$-th $\mathrm{BS}$ can be written as

$$
\mathbf{x}_{n l}(t)=\mathbf{w}_{n l} s_{l}(t),
$$

where $s_{l}(t) \in \mathbb{C}$ represents the information symbol at time $t$ and $\mathbf{w}_{n l} \in \mathbb{C}^{M}$ is the unnormalised transmit beamforming vector for user $l$ at $n$-th BS. Without loss of generality we assume that $s_{l}(t)$ is normalised such that $\mathbb{E}\left\{\left|s_{l}(t)\right|^{2}\right\}=1$ and that all data streams are independent such that $\mathbb{E}\left\{s_{l}(t) s_{j}(t)^{*}\right\}=0$ if $l \neq i$. In this paper, we assume perfect channel state information (CSI) knowledge at both the transmitter and the receiver. We denote the MIMO channel between the $n$-th BS and the global user $g$ as $\mathbf{H}_{n g} \in \mathbb{C}^{N_{r} \times M}$, where $N_{r}$ is the number of receive antennas at the global user. The intended signal at the global user is given by

$$
\mathbf{r}=\mathbf{H}_{1 g} \mathbf{w}_{1 g_{1}} s g_{1}(n)+\mathbf{H}_{2 g} \mathbf{w}_{2 g_{2}} s g_{2}(n)
$$

The global user deploys the receive beamformers $\mathbf{u}_{1}$ and $\mathbf{u}_{2}$. By using SVD, the channel matrices between the BSs can be written as

$$
\begin{aligned}
& \mathbf{H}_{1 g}=\mathbf{U}_{1} \boldsymbol{\Lambda}_{1} \mathbf{V}_{1}^{H}, \\
& \mathbf{H}_{2 g}=\mathbf{U}_{2} \boldsymbol{\Lambda}_{2} \mathbf{V}_{2}^{H},
\end{aligned}
$$

where $\mathbf{U}_{1} \in \mathbb{C}^{N_{r} \times N_{r}}$ (respectively $\mathbf{U}_{2} \in \mathbb{C}^{N_{r} \times N_{r}}$ ) and $\mathbf{V}_{1} \in \mathbb{C}^{M \times M}$ (respectively $\mathbf{V}_{2} \in \mathbb{C}^{M \times M}$ ) are the unitary matrices and $\boldsymbol{\Lambda}_{1} \in \mathbb{C}^{N_{r} \times M}$ (respectively $\boldsymbol{\Lambda}_{2} \in \mathbb{C}^{N_{r} \times M}$ ) is the diagonal matrix of the singular values of $\mathbf{H}_{1 g}$ (respectively $\mathbf{H}_{2 g}$ ) sorted in descending order. The SVD of the MIMO channels allows us to represent the global user as two virtual users denoted as $g_{1}$ and $g_{2}$. Denote $\mathbf{u}_{1}$ and $\mathbf{u}_{2}$ as the singular vectors corresponding to the largest singular values of $\mathbf{H}_{1 g}$ and $\mathbf{H}_{2 g}$ respectively. The decomposed received signal at the virtual users $g_{1}$ and $g_{2}$ can be written as

$$
\begin{aligned}
y_{g_{1}} & =\mathbf{u}_{1}^{H}\left[\mathbf{H}_{1 g} \mathbf{w}_{1 g} s g_{1}+\mathbf{H}_{2 g} \mathbf{w}_{2 g} s g_{2}+\mathbf{H}_{1 g}\left(\mathbf{w}_{11} s_{1}+\mathbf{w}_{12} s_{2}\right)\right. \\
& \left.+\mathbf{H}_{2 g}\left(\mathbf{w}_{23} s_{3}+\mathbf{w}_{24} s_{4}\right)+n_{g_{1}}\right] \\
y_{g_{2}} & =\mathbf{u}_{2}^{H}\left[\mathbf{H}_{2 g} \mathbf{w}_{2 g} s g_{2}+\mathbf{H}_{1 g} \mathbf{w}_{1 g} s g_{1}+\mathbf{H}_{2 g}\left(\mathbf{w}_{23} s_{3}+\mathbf{w}_{24} s_{4}\right)\right. \\
& \left.+\mathbf{H}_{1 g}\left(\mathbf{w}_{11} s_{1}+\mathbf{w}_{12} s_{2}\right)+n_{g_{2}}\right]
\end{aligned}
$$

respectively. Lets us denote the effective channel vector between the $n$-th BS and the virtual user $g_{v}$ as $\mathbf{q}_{n, g_{v}}$. The effective channels between the BSs and the virtual users can be written as

$$
\begin{aligned}
& \mathbf{q}_{11}=\mathbf{u}_{1}^{H} \mathbf{H}_{1 g}, \\
& \mathbf{q}_{12}=\mathbf{u}_{2}^{H} \mathbf{H}_{1 g}, \\
& \mathbf{q}_{21}=\mathbf{u}_{1}^{H} \mathbf{H}_{2 g}, \\
& \mathbf{q}_{22}=\mathbf{u}_{2}^{H} \mathbf{H}_{2 g} .
\end{aligned}
$$

\section{B. System Metric Design}

All the users have specific data rate requirements in order to establish successful connections. Let us denote a set of local users belonging to the $n$-th $\mathrm{BS}$ as $\mathcal{L}_{n} \subset \mathcal{L}$. Let us define the correlation matrix of the channel from the $n$-th BS to $l$-th local user as $\mathbf{R}_{n l}=\left[\mathbf{h}_{n l} \mathbf{h}_{n l}^{H}\right]$. The correlation matrix of the channel from the $n$-th BS to the virtual user $g_{v}$ is denoted as $\mathbf{G}_{n g_{v}}=\left[\mathbf{q}_{n g_{v}}^{H} \mathbf{q}_{n g_{v}}\right]$. The intracell and intercell interference powers experienced by the $l$-th local user are given as

$$
\begin{aligned}
& I_{n}= \sum_{\substack{i=1 \\
i \neq l}}^{L_{n}} \mathbf{w}_{n i}^{H} \mathbf{R}_{n l} \mathbf{w}_{n i}+\mathbf{w}_{1 g_{1}}^{H} \mathbf{R}_{n l} \mathbf{w}_{1 g_{1}}, \\
& I_{p}=\sum_{\substack{j=1 \\
p \neq n}}^{L_{p}} \mathbf{w}_{p j}^{H} \mathbf{R}_{p l} \mathbf{w}_{p j}+\mathbf{w}_{2 g_{2}}^{H} \mathbf{R}_{p l} \mathbf{w}_{2 g_{2}},
\end{aligned}
$$

respectively. The downlink SINR of the $l$-th local user at $n$-th $\mathrm{BS}$ is given by

$$
\operatorname{SINR}_{l}^{n}=\frac{\mathbf{w}_{n l}^{H} \mathbf{R}_{n l} \mathbf{w}_{n l}}{I_{n}+I_{p}+\sigma_{l}^{2}} .
$$

where $\mathbf{h}_{n l} \in \mathbb{C}^{M \times 1}$ be channel vector between the $n$-th BS and the $l$-th local user, and $\sigma^{2}$ is the noise variance at the $l$-th local user. Respectively, the SINR of the virtual users $g_{v 1}$ and $g_{v 2}$ are given by

$$
\begin{aligned}
& \operatorname{SINR}_{g_{1}}=\frac{\mathbf{w}_{1 g_{1}}^{H} \mathbf{G}_{11} \mathbf{w}_{1 g_{1}}}{\sum_{i=1}^{L_{1}} \mathbf{w}_{1 i}^{H} \mathbf{G}_{11} \mathbf{w}_{1 i}+\sum_{j=1}^{L_{2}} \mathbf{w}_{2 j}^{H} \mathbf{G}_{21} \mathbf{w}_{2 j}+\sigma_{g_{1}}^{2}} . \\
& \operatorname{SINR}_{g_{2}}=\frac{\mathbf{w}_{2 g_{2}}^{H} \mathbf{G}_{22} \mathbf{w}_{2 g_{2}}}{\sum_{i=1}^{L_{1}} \mathbf{w}_{1 i}^{H} \mathbf{G}_{12} \mathbf{w}_{1 i}+\sum_{j=1}^{L_{2}} \mathbf{w}_{2 j}^{H} \mathbf{G}_{22} \mathbf{w}_{2 j}+\sigma_{g_{2}}^{2}} .
\end{aligned}
$$

Therefore, the total data rate of the global user is given by

$$
R_{g}=R_{g 1}+R_{g 2}=\log _{2}\left(1+\operatorname{SINR}_{g_{1}}\right)+\log _{2}\left(1+\operatorname{SINR}_{g_{2}}\right) \text {. }
$$

\section{SVD BASED BEAMFORMING DESIGN}

We aim to operate with the minimum total transmission power that will guarantee all the users their specific data rate target. We denote the specific data rate for the $l$-th local user and the global user as $r_{l}$ and $r_{g}$ respectively. Our optimization problem is formulated as

$$
\begin{aligned}
\min & \sum_{n \in \mathcal{N}} \sum_{l \in \mathcal{L}}\left\|\mathbf{w}_{n l}\right\|_{2}^{2}+\left\|\mathbf{w}_{1 g_{1}}\right\|_{2}^{2}+\left\|\mathbf{w}_{2 g_{2}}\right\|_{2}^{2}, \\
\text { s.t } \quad \log _{2}\left(1+\mathrm{SINR}_{l}\right) \geq r_{l}, \quad \forall l & \\
& R_{g} \geq r_{g} .
\end{aligned}
$$


In [4], it was proved that at optimality, the constraints in (17) will be satisfied with equality. For analysis purpose, we convert the data rates in (17) to SINRs. The local user SINR is determined as $\operatorname{SINR}_{l}=2^{r_{l}}-1$. By setting the data rate at virtual user $g 1$ as a variable $\theta$, where $0 \leq \theta \leq r_{g}$, the SINRs of the virtual users $g_{1}$ and $g_{2}$ can be written as $\operatorname{SINR}_{g_{1}}=2^{\theta}-1$ and $\operatorname{SINR}_{g_{2}}=2^{\left(r_{g}-\theta\right)}-1$, respectively. Respectively, let us define the total interference experienced by the $l$-th local user and virtual users $g_{1}$ and $g_{2}$ as

$$
\begin{aligned}
I_{l}= & \sum_{n \in \mathcal{N}} \sum_{k \neq l} \mathbf{w}_{p k}^{H} \mathbf{R}_{n k} \mathbf{w}_{p k}+\mathbf{w}_{1 g_{1}}^{H} \mathbf{R}_{p g_{1}} \mathbf{w}_{1 g_{1}} \\
& +\mathbf{w}_{2 g_{2}}^{H} \mathbf{R}_{2 g_{2}} \mathbf{w}_{2 g_{2}}, \\
I_{g_{1}}= & \sum_{n \in \mathcal{N}} \sum_{l \in \mathcal{L}} \mathbf{w}_{n l}^{H} \mathbf{R}_{n l} \mathbf{w}_{n l}+\mathbf{w}_{2 g_{2}}^{H} \mathbf{G}_{1 g_{2}} \mathbf{w}_{2 g_{2}}, \\
I_{g_{2}}= & \sum_{n \in \mathcal{N}} \sum_{l \in \mathcal{L}} \mathbf{w}_{n l}^{H} \mathbf{R}_{n l} \mathbf{w}_{n l}+\mathbf{w}_{1 g_{1}}^{H} \mathbf{G}_{2 g_{1}} \mathbf{w}_{1 g_{1}} .
\end{aligned}
$$

Given the SINR thresholds of the $l$-th local user and the virtual user $g_{v}$ as $\gamma_{l}$ and $\gamma_{g_{v}}$, we rewrite (17) as

$$
\begin{aligned}
\min & \sum_{n \in \mathcal{N}} \sum_{l \in \mathcal{L}} \mathbf{w}_{n l}^{H} \mathbf{w}_{n l}+\mathbf{w}_{1 g_{1}}^{H} \mathbf{w}_{1 g_{1}}+\mathbf{w}_{2 g_{2}}^{H} \mathbf{w}_{2 g_{2}} \\
\text { s.t } & \mathbf{w}_{n l}^{H} \mathbf{R}_{n l} \mathbf{w}_{n l}-\gamma_{l} I_{l} \geq \gamma_{n l} \sigma_{l}^{2}, \quad \forall l, \\
& \mathbf{w}_{1 g_{1}}^{H} \mathbf{G}_{1 g_{1}} \mathbf{w}_{1 g_{1}}-\gamma_{g_{1}} I_{g_{1}} \geq \gamma_{g_{1}} \sigma_{g_{1}}^{2}, \quad \forall l, \\
& \mathbf{w}_{2 g_{2}}^{H} \mathbf{G}_{2 g_{2}} \mathbf{w}_{2 g_{2}}-\gamma_{g_{2}} I_{g_{2}} \geq \gamma_{g_{2}} \sigma_{g_{2}}^{2}, \quad \forall l .
\end{aligned}
$$

The constraints set in (17) makes the whole problem nonconvex but after necessary manipulation, the problem can be convexified. Let us denote $\mathbf{W}_{n l}=\mathbf{w}_{n l} \mathbf{w}_{n l}^{H}, \mathbf{W}_{1 g_{1}}=\mathbf{w}_{1 g_{1}} \mathbf{w}_{1 g}^{H}$ and $\mathbf{W}_{2 g_{2}}=\mathbf{w}_{2 g_{2}} \mathbf{w}_{2 g}^{H}$. We then use the rule $\mathbf{w}^{H} \mathbf{R} \mathbf{w}=$ $\operatorname{Tr}\left[\mathbf{R} \mathbf{w}^{H} \mathbf{w}\right]=\operatorname{Tr}[\mathbf{R W}]$ to rewrite the (18)-(21) as

$$
\begin{aligned}
& I_{l}= \sum_{n \in \mathcal{N}} \sum_{k \neq l} \operatorname{Tr}\left[\mathbf{R}_{n k} \mathbf{W}_{p k}\right]+\operatorname{Tr}\left[\mathbf{G}_{1 g_{1}} \mathbf{W}_{1 g_{1}}\right] \\
&+\operatorname{Tr}\left[\mathbf{G}_{2 g_{2}} \mathbf{W}_{2 g_{2}}\right], \\
& I_{g_{1}}= \sum_{n \in \mathcal{N}} \sum_{l \in \mathcal{L}} \operatorname{Tr}\left[\mathbf{R}_{n l} \mathbf{W}_{n l}\right]+\operatorname{Tr}\left[\mathbf{G}_{2 g_{2}} \mathbf{W}_{2 g_{2}}\right] \\
& I_{g_{2}}= \sum_{n \in \mathcal{N}} \sum_{l \in \mathcal{L}} \operatorname{Tr}\left[\mathbf{R}_{n l} \mathbf{W}_{n l}\right]+\operatorname{Tr}\left[\mathbf{G}_{1 g_{1}} \mathbf{W}_{1 g_{1}}\right], \\
& \min \sum_{n \in \mathcal{N}} \sum_{l \in \mathcal{L}} \operatorname{Tr}\left[\mathbf{W}_{n l}\right]+\operatorname{Tr}\left[\mathbf{W}_{1 g_{1}}\right]+\operatorname{Tr}\left[\mathbf{W}_{2 g_{2}}\right] \\
& \text { s.t } \operatorname{Tr}\left[\mathbf{R}_{n l} \mathbf{W}_{n l}\right]-\gamma_{l} I_{l} \geq \gamma_{n l} \sigma_{l}^{2}, \quad \forall l, \\
& \operatorname{Tr}\left[\mathbf{G}_{1 g_{1}} \mathbf{W}_{1 g_{1}}\right]-\gamma_{g_{1}} I_{g_{1}} \geq \gamma_{g_{1}} \sigma_{g_{1}}^{2}, \quad \forall l, \\
& \operatorname{Tr}\left[\mathbf{G}_{2 g_{2}} \mathbf{W}_{2 g_{2}}\right]-\gamma_{g_{2}} I_{g_{2}} \geq \gamma_{g_{2}} \sigma_{g_{2}}^{2}, \quad \forall l, \\
& \mathbf{W}_{n l} \succeq 0, \quad \mathbf{W}_{n l}=\mathbf{W}_{n l}^{H}, \quad \operatorname{rank}\left[\mathbf{W}_{n l}\right]=1, \quad \forall n, \forall l \\
& \mathbf{W}_{1 g_{1}} \succeq 0, \quad \mathbf{W}_{1 g_{1}}=\mathbf{W}_{1 g_{1}}^{H}, \quad \operatorname{rank}\left[\mathbf{W}_{1 g_{1}}\right]=1 \\
& \mathbf{W}_{2 g_{2}} \succeq 0, \quad \mathbf{W}_{2 g_{2}}=\mathbf{W}_{2 g_{2}}^{H}, \quad \operatorname{rank}\left[\mathbf{W}_{2 g_{2}}\right]=1
\end{aligned}
$$

where, $\mathbf{W} \succeq 0$ means $\mathbf{W}$ positive semidefinite. The ranks of $\left\{\mathbf{W}_{n l}\right\}_{\forall n, \forall l}, \mathbf{W}_{1 g_{1}}$, and $\mathbf{W}_{2 g_{2}}$ are nonconvex. Nevertheless, relaxing all the rank constraints gives the following relaxed semidefinite optimization problem [5]

$$
\begin{aligned}
\min & \sum_{n \in \mathcal{N}} \sum_{l \in \mathcal{L}} \operatorname{Tr}\left[\mathbf{W}_{n l}\right]+\operatorname{Tr}\left[\mathbf{W}_{1 g_{1}}\right]+\operatorname{Tr}\left[\mathbf{W}_{2 g_{2}}\right] \\
\text { s.t } & \operatorname{Tr}\left[\mathbf{R}_{n l} \mathbf{W}_{n l}\right]-\gamma_{l} I_{l} \geq \gamma_{n l} \sigma_{l}^{2}, \quad \forall l, \\
& \operatorname{Tr}\left[\mathbf{G}_{1 g_{1}} \mathbf{W}_{1 g_{1}}\right]-\gamma_{g_{1}} I_{g_{1}} \geq \gamma_{g_{1}} \sigma_{g_{1}}^{2}, \quad \forall l, \\
& \operatorname{Tr}\left[\mathbf{G}_{2 g_{2}} \mathbf{W}_{2 g_{2}}\right]-\gamma_{g_{2}} I_{g_{2}} \geq \gamma_{g_{2}} \sigma_{g_{2}}^{2}, \quad \forall l, \\
& \mathbf{W}_{n l} \succeq 0, \quad \mathbf{W}_{n l}=\mathbf{W}_{n l}^{H}, \forall n, \forall l, \\
& \mathbf{W}_{1 g_{1}} \succeq 0, \quad \mathbf{W}_{1 g_{1}}=\mathbf{W}_{1 g_{1}}^{H}, \\
& \mathbf{W}_{2 g_{2}} \succeq 0, \quad \mathbf{W}_{2 g_{2}}=\mathbf{W}_{2 g_{2}}^{H},
\end{aligned}
$$

which can be solved to an arbitrary accuracy using SDP solvers like YALMIP [17]. We note that if the (26) is feasible, it will provide rank-1 matrices $\left\{\mathbf{W}_{n l}\right\}_{\forall n, \forall l}, \mathbf{W}_{1 g_{1}}$, and $\mathbf{W}_{2 g_{2}}$ [5], [18]. However, if the rank of $\left\{\mathbf{W}_{n l}^{\star}\right\}_{\forall n, \forall l}, \mathbf{W}_{1 g_{1}}^{\star}$, and $\mathbf{W}_{2 g_{2}}^{\star}$ are greater than one, we can use the randomization techniques to heuristically find the $\mathbf{w}_{n l}, \forall n, \forall l, \mathbf{w}_{1 g_{1}}$, and $\mathbf{w}_{2 g_{2}}$ [18]. Note that if rank of $\left\{\mathbf{W}_{n l}^{\star}\right\}_{\forall n, \forall l}, \mathbf{W}_{1 g_{1}}^{\star}$, and $\mathbf{W}_{2 g_{2}}^{\star}$ are greater than one, then the heuristic $\left\{\mathbf{w}_{n l}\right\} \forall n, \forall l, \mathbf{w}_{1 g_{1}}$, and $\mathbf{w}_{2 g_{2}}$ will provide a lower bound for the minimum required transmission power. Apparently, (26) is a dual of a dual program (i.e., bidual) of (25) [19], [20].

\section{NUMERICAL EXAMPLE}

We consider a multicell multiuser network with two BSs and five users. Each BS is equipped with $M=5$ antennae and it serves two single antenna local users. A global user is equipped with two receive antennae and it is served by both BSs. All BSs operate on the same frequency henceforth we assume all users experience significant intra-cell and inter-cell interference. Each user has a specific data rate target which needs to be satisfied for a successful connection. The channel vectors $\mathbf{h}_{n l}$ and $\mathbf{H}_{n g}$ were generated as i.i.d Gaussian random variables and the noise variance was set to $\sigma^{2}=1$ for all users. The random channels are generated between users and all BSs with zero mean and unity variance. The data rate targets for a pair of local users at each BS were set to $1.5 \mathrm{bits} / \mathrm{s} / \mathrm{Hz}$ and 2 bits/s/Hz respectively. The data rate target for the global user was set to $4 \mathrm{bits} / \mathrm{s} / \mathrm{Hz}$.

Subfigure 2a shows the total transmission power, for a single channel realization, when the data rate from BS1 to the global user is varied from 0 to $4 \mathrm{bits} / \mathrm{s} / \mathrm{Hz}$ with step size $\delta=0.1$ bits/s/Hz. We observe that the minimum total transmission power is achieved when BS1 contribute $2.6 \mathrm{bits} / \mathrm{s} / \mathrm{Hz}$ of the 4 $\mathrm{bits} / \mathrm{s} / \mathrm{Hz}$. It is possible that, for a given channel realization, all the data rate to the global user comes from only one BS. In subfigure $2 \mathrm{~b}$, we study the average data rate contributed by BS1 to the global user over 250 random channel realizations. As anticipated, we note that on average, BS1 will contribute 2 $\mathrm{bits} / \mathrm{s} / \mathrm{Hz}$, whereas the remaining data rate will be contributed by BS2.

\section{CONCLUSion}

We studied a multicell multiuser network which simultaneously considers coordinated beamforming and joint transmission. The network consists of single antenna local users and 

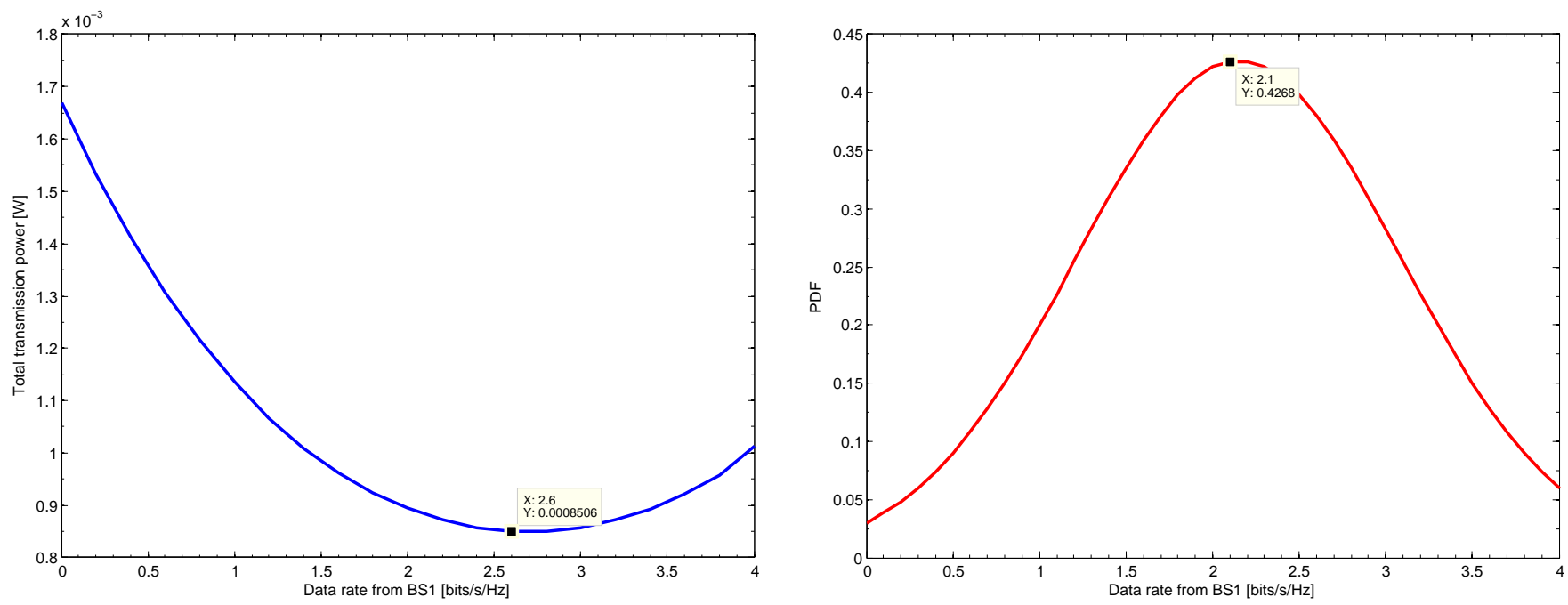

(a) Total transmission power for one channel realization.

(b) Average achievable data rate at the global user contributed by BS1.

Fig. 2. Performance analysis of the proposed SVD based beamformer.

one multi-antenna global user. The global user is served by more than one BS, whereas the local users are assigned to only one BS at a time. We considered beamforming design using power minimization criterion. The simulation results concluded that on average, the minimum total transmission power will be achieved if the data rate to the global user is equally shared between the BSs.

\section{REFERENCES}

[1] E. Björnson and E. Jorswieck, "Optimal Resource Allocation in Coordinated Multi-Cell Systems," Foundation and Trends in Communications and Information Theory, vol. 9, no. 2012, pp. 113-381, 2013.

[2] N. Bhushan, J. Li, D. Malladi, R. Gilmore, D. Brenner, A. Damnjanovic, and R. Teja, "Network Densification: The Dominant Theme for Wireless Evolution into 5G," IEEE Communications Magazine, no. February, pp. 82-89, Feb. 2014.

[3] F. Rashid-Farrokhi, K. J. R. Liu, and L. Tassiulas, "Transmit Beamforming and Power Control for Cellular Wireless Systems," IEE Journal on selected areas in communications, vol. 16, no. 8, pp. 1437-1450, Oct. 1998.

[4] M. Schubert and H. Boche, "Solution of the Multiuser Downlink Beamforming Problem With Individual SINR Constraints," IEEE Transactions on Vehicular Technology, vol. 53, no. 1, pp. 18-28, Jan. 2004.

[5] M. Bengtsson and B. Ottersten, "Optimal Downlink Beamforming Using Semidefinite Optimization," in In proceedings of 37th Annual Allerton Conference on Communication, Stockholm, 1999, pp. 987-996.

[6] D. W. H. Cai, T. Q. S. Quek, C. W. Tan, and S. H. Low, "MaxMin SINR Coordinated Multipoint Downlink Transmission Duality and Algorithms," IEEE Transactions on Signal Processing, vol. 60, no. 10, pp. 5384-5395, Oct. 2012.

[7] D. Senaratne and C. Tellambura, "Generalized Singular Value Decomposition for Coordinated Beamforming in MIMO Systems," in IEEE Globecom 2010 proceedings, 2010, pp. 0-5.

[8] K. Dawui and D. Slock, "Multiuser-MIMO Downlink TX-RX Design Based on SVD Channel Diagonalization and Multiuser Diversity," in Conference Record of the Thirty-Ninth Asilomar Conference onSignals, Systems and Computers, 2005., no. 1, 2005, pp. 1493-1497.

[9] H. Dahrouj and W. Yu, "Coordinated Beamforming for the Multicell Multi-antenna Wireless System," IEEE Transactions on Wireless Communications, vol. 9, no. 5, pp. 1748-1759, May 2010
[10] E. Björnson, M. Kountouris, and M. Debbah, "Massive MIMO and Small Cells: Improving Energy Efficiency by Optimal Soft-Cell Coordination," in 2013 20th International Conference on Telecommunications, ICT 2013, 2013, pp. 1-5.

[11] Y. Rahulamathavan, S. Member, and K. Cumanan, "A Mixed SINRBalancing and SINR-Target-Constraints-Based Beamformer Design Technique for Spectrum-Sharing Networks," IEEE Transactions on Vehicular Technology, vol. 60, no. 9, pp. 4403-4414, 2011.

[12] U.-d. M.-m. S. Duality, S. He, Y. Huang, and L. Yang, "A Multi-Cell Beamforming Design by," IEEE Transactions on Wireless Communications, vol. 11, no. 8, pp. 2858-2867, 2012.

[13] J. Zhao, T. Q. S. Quek, S. Member, Z. Lei, and S. Member, "Coordinated Multipoint Transmission with Limited Backhaul Data Transfer," IEEE Transactions on Wireless Communications, vol. 12, no. 6, pp. 27622775, 2013.

[14] Y. Wu, G. Bournaka, and S. Lambotharan, "Coordinated Beamforming with Mixed SINR-Balancing and SINR-Target-Constraints for Multicell Wireless Networks," in IEEE WCNC, vol. 1, no. 1, 2014, pp. 908-912.

[15] B. Basutli and S. Lambotharan, "Distributed Beamformer Design Under Mixed SINR Balancing and SINR-Target-Constraints," in IEEE International Conference on Digital Signal Processing, Singapore, 2015, pp. 530-534.

[16] G. Bournaka, Y. Rahulamathavan, K. Cumanan, S. Lambotharan, and F. Lazarakis, "Base station beamforming technique using multiple signal-to-interference plus noise ratio balancing criteria," IET Signal Process, vol. 9, no. August 2014, pp. 248-259, 2015.

[17] J. Efberg, "YALMIP : A toolbox for modeling and optimization in MATLAB," in IEEE International Symposium on Computer Aided Control Sysytems Design, Tapei, 2004, pp. 284-289.

[18] Z.-q. Luo, A. M.-c. So, Y. Ye, and S. Zhang, "Semidefinite Relaxation of Quadratic Optimization Problems," IEEE Signal Processing Magazine, no. May, pp. 20-34, May 2010.

[19] R. T. Rockafella, Conjugate Duality and Optimization. SIAM, 1974.

[20] S. Boyd and L. Vandenberghe, Convex Optimization, 3rd ed. University Press, Cambridge UK, 2004, vol. 25. 